\title{
Plasma lipoxin A4 levels in childhood chronic spontaneous urticaria
}

\author{
Fatih Dilek ${ }^{1}$, Deniz Özçeker ${ }^{2}$, Eray Metin Güler ${ }^{3}$, Emin Özkaya ${ }^{4}$, Mebrure Yazıc1 $^{4}$, Zeynep \\ Tamay $^{5}$, Abdurrahim Koçyiğit ${ }^{3}$, Nermin Güler ${ }^{6}$ \\ ${ }^{1}$ Department of Pediatric Allergy and Immunology, University of Health Sciences, Kayseri Training and Research Hospital, \\ Kayseri; ${ }^{2}$ Department of Pediatric Allergy, University of Health Sciences, Okmeydanı Training and Research Hospital; \\ Departments of ${ }^{3}$ Clinical Biochemistry, ${ }^{4}$ Pediatric Allergy and Immunology, Bezmialem Vakif University Medical Faculty; \\ ${ }^{5}$ Department of Pediatric Allergy, Istanbul University Istanbul Medical Faculty; ${ }^{6}$ Department of Pediatrics, Istanbul Bilim \\ University, İstanbul, Turkey.E-mail: drfatihdilek@yahoo.com
}

Received: 12th October 2017, Accepted: 9th December 2017

SUMMARY: Dilek F, Özçeker D, Güler EM, Özkaya E, Yazıcı M, Tamay Z, Koçyiğit A, Güler N. Plasma lipoxin A4 levels in childhood chronic spontaneous urticaria. Turk J Pediatr 2018; 60: 527-534.

Chronic spontaneous urticaria (CSU) is an idiopathic inflammatory disorder. Despite great research progress, the pathogenesis of the disease is still not fully understood. Lipoxins (LXs) are autacoid lipid metabolites that are the first discovered members of a new genus named called "specialized proresolving mediators". In this study, we aimed to investigate the possible role of $\mathrm{LXA}_{4}$ in the pathogenesis of CSU.

Forty-two children with CSU and 25 healthy children were enrolled in the study. The demographic and clinical features of patients were evaluated, autologous serum skin tests (ASSTs), and routine laboratory assessments were performed. Disease activity was determined using the urticaria activity score. An enzyme-linked immunosorbent assay was used to evaluate LXA $_{4}$ plasma levels.

The median value of plasma $\mathrm{LXA}_{4}$ was found to be $60.8 \mathrm{ng} / \mathrm{ml}$ (interquartile range, 48.1-71.8) in CSU patients and $137.4 \mathrm{ng} / \mathrm{ml}(121.4-150.8)$ in the control group. The difference between the groups was statistically significant $(\mathrm{p}<0.001)$. Additionally, the median plasma $\mathrm{LXA}_{4}$ levels in the ASST-positive patients were significantly reduced compared to the ASST-negative ones (45.8 [36.7-67.6] versus 63.8 [58.3-78.9] $\mathrm{ng} / \mathrm{ml}$, respectively, $\mathrm{p}<0.05)$.

Our results showed that diminished $\mathrm{LXA}_{4}$ biosynthesis may be a critical part of CSU pathogenesis in children, especially in patients with an autoimmune component.

Key words: chronic spontaneous urticaria, children, lipoxin A4, disease severity.

Chronic urticaria is an inflammatory disorder of the skin characterized by the presence of the daily or near daily appearance of urticaria (hives) with/or without angioedema for more than six weeks. ${ }^{1}$ The disease has two subtypes-chronic inducible urticaria and chronic spontaneous urticaria (CSU). ${ }^{2}$ If an urticarial event is triggered after a physical stimulus, such as cold, a vibration, pressure, or water contact, it constitutes chronic inducible urticaria. If the urticarial event is not associated with a physical stimulus, it is classified as CSU. ${ }^{2}$
Despite much research in last few decades, the etiopathogenesis of CSU is still not fully understood. Although some controversy exists, there is strong evidence of an underlying autoimmune mechanism in $30 \%-40 \%$ of CSU patients. ${ }^{3}$ It is well known that persistent and uncontrolled mast cell activation is the hallmark of disease. ${ }^{4,5}$ Although the cause of this phenomenon is unknown, it is thought that the proinflammatory cytokines released from the inflammatory cells that migrate to the dermis decrease the sensitivity threshold of the mast cells for degranulation. ${ }^{5}$ 
Acute inflammation is an innate immune response to pathogens, toxins, and injuries and is vital, protective, and useful.6,7 The best end-point of this process is complete resolution. If this does not happen, uncontrolled inflammation can cause chronic inflammation, tissue injury, and scarring. ${ }^{8}$ Recent studies have clearly shown that the resolution of inflammation is a tightly regulated, active course shaped by a family of lipid mediators called "specialized proresolving lipid mediators (SPMs)". ${ }^{7}$ The defined members of this family are lipoxins (LXs), protectins, resolvins, and maresins. ${ }^{8}$

LXs are the first discovered proresolving eicosanoids that are synthesized from arachidonic acid via lipoxygenases with different pathways in various cells, including neutrophils, eosinophils, platelets, alveolar macrophages, and airway epithelial cells. ${ }^{7,}{ }^{9-11}$ LXA $_{4}$ exerts its bioactions mainly through the agonism of a $\mathrm{G}$ protein-coupled receptor called formyl peptide receptor-2 (FPR2/ALX), in addition to the agonism of the aryl hydrocarbon receptor and the partial antagonism of the cysteinyl leukotriene receptor 1.9,12-14 FPR2/
ALX is expressed on a number of cells, including polymorphonuclear cells, eosinophils, monocytes/macrophages, B-lymphocytes, fibroblasts, enterocytes, airway epithelia, and vascular smooth muscle cells.9,13,15-18

LXA $_{4}$ and its analogues have potent antiinflammatory and immunoregulatory features. They inhibit eosinophil and neutrophil chemotaxis, vascular adhesion, transendothelial migration, reactive oxygen species (ROS) generation, and azurophilic degranulation of neutrophils. ${ }^{7} \mathrm{LXA}_{4}$ reduces nuclear factor (NF)$\kappa \mathrm{B}$ activation and the release of inflammatory cytokines. ${ }^{7}$ It also directly influences vascular endothelia, inhibits ROS production, decreases the expression of adhesion molecules and vascular permeability. 7,13,19

Direct and indirect evidence obtained from recent studies has shown that LXs play prominent roles in the pathogenesis of various disorders, such as cystic fibrosis, acute lung injury, sepsis, peritonitis, rheumatoid arthritis, and Alzheimer's disease, and in some allergic diseases, such as asthma, allergic rhinitis, atopic dermatitis, and exercise-induced bronchoconstriction. ${ }^{7,20-23}$ Despite all its

Table I. Demographic and Clinical Features of the Study Group.

\begin{tabular}{lc}
\hline & $\begin{array}{c}\text { CSU patients } \\
(\mathrm{n}=42)\end{array}$ \\
\hline Age in years (mean, $\pm \mathrm{SD})$ & $11.0 \pm 4.2$ \\
Gender $(\mathrm{M} / \mathrm{F})$ & $25 / 17$ \\
Angioedema $(\mathrm{n}[\%])$ & $16(38 \%)$ \\
Total Ig E $>100 \mathrm{I} \mu / \mathrm{L}(\mathrm{n}[\%])$ & $12(29 \%)$ \\
Eosinophils $>5 \%(\mathrm{n}[\%])$ & $3(7 \%)$ \\
Aeroallergen sensitization (n[\%]) & $5 / 32(16 \%)$ \\
ASST positivity (n[\%]) & \\
$\quad$ ASST-positive & $10 / 32(31 \%)$ \\
ASST-negative & $22 / 32(69 \%)$ \\
UAS (median, [IQR]) & $3.0(2.8-5)$ \\
Treatment (n[\%]) & \\
$\quad$ Second-generation antihistamines at licensed doses & $28(67 \%)$ \\
Second-generation antihistamines at high doses & $4(10 \%)$ \\
Combination of second-generation antihistamines + montelukast & $10(23 \%)$ \\
\hline
\end{tabular}

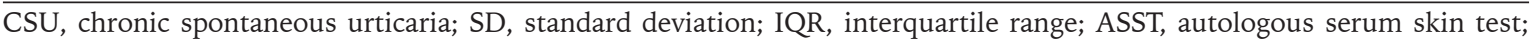
UAS, urticaria activity score. 


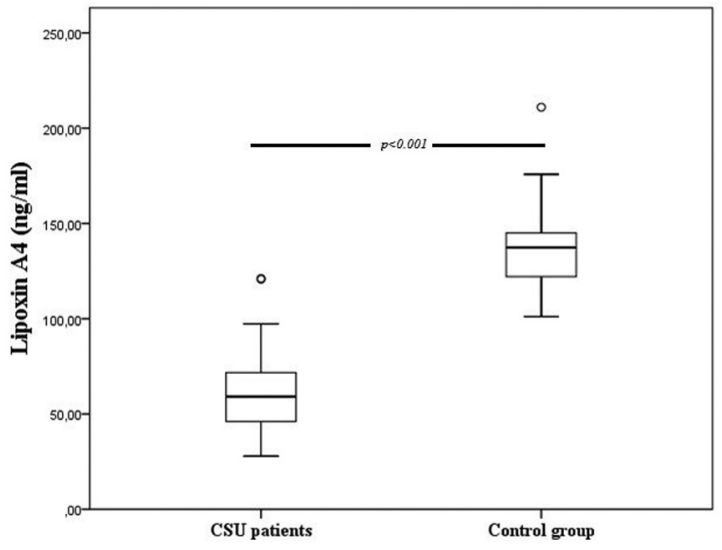

Fig. 1. Plasma $\mathrm{LXA}_{4}$ levels in chronic spontaneous urticaria (CSU) patients and control group participants.

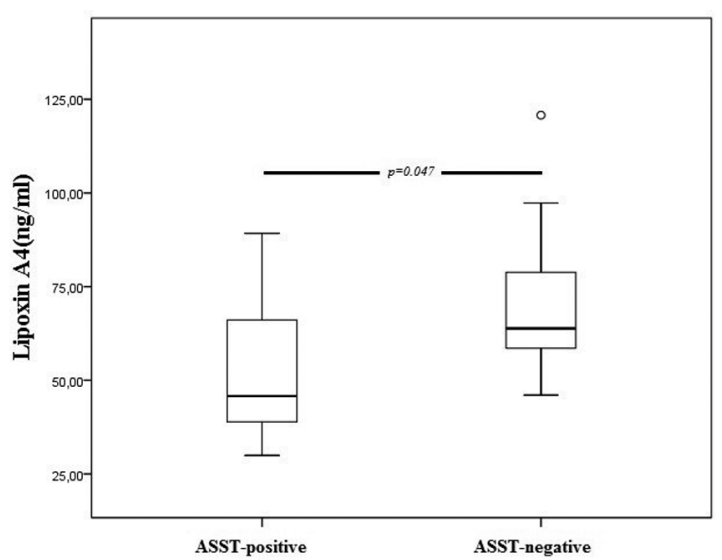

Fig. 2. Plasma LXA 4 levels in autologous serum skin test (ASST)-positive and -negative patients.

extraordinarily promising bioactivities, the possible role of $\mathrm{LXA}_{4}$ in the pathogenesis of CSU has not been investigated until now. Therefore, we hypothesize that inadequate LX biosynthesis in patients with CSU may cause a defect in the resolution of dermal inflammation that leads to chronic, uncontrolled inflammation and that this phenomenon may be an unresolved aspect of the pathogenesis of CSU.

\section{Material and Methods \\ Patients}

This study was conducted between April 2016 and July 2016 in outpatient clinics at two university hospitals in the same city. A total of 58 pediatric CSU patients who were admitted during the previous six months were invited to join the study. Patients who were diagnosed with

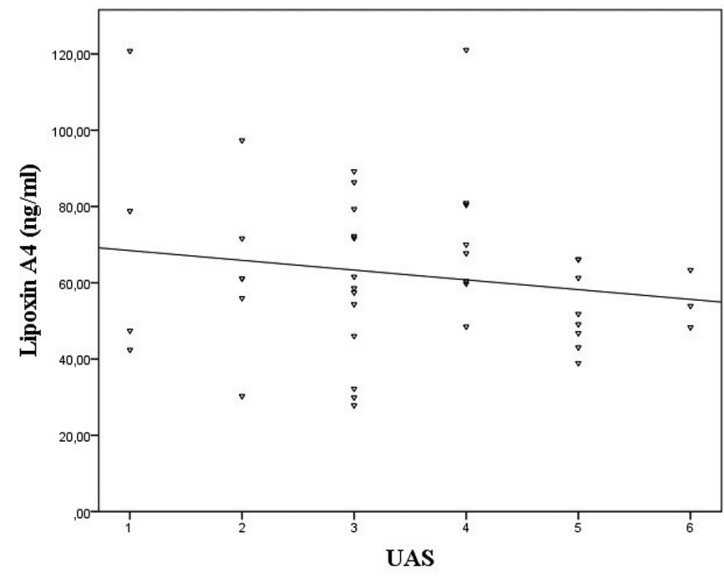

Fig.3. Scatter graph showing the $\mathrm{LXA}_{4}$ and urticaria activity score (UAS) relationship in patients with chronic spontaneous urticaria (CSU).

chronic inducible urticaria and for whom the underlying causes of CSU had been identified (outside of autoimmunity) were excluded from the study. Diagnosis and classification were based on the European Academy of Allergy and Clinical Immunology guidelines. ${ }^{2}$ Children with diseases or conditions that can potentially alter plasma $\mathrm{LXA}_{4}$ levels, including concomitant diagnosed atopic dermatitis, allergic rhinitis, asthma, or any other chronic disease, or children who are taking any other drugs or nutritional supplements (e.g., fish oil) except antihistaminics or montelukast were excluded from the study. After all exclusions, 42 patients with CSU were finally enrolled in the study.

The control group consisted of 25 healthy children who were periodically attending pediatric welfare clinics in the same hospitals for regular checkups. These children had no history, signs, or symptoms of acute or chronic diseases and were not taking any medications or nutritional supplements. The study was performed in accordance with the tenets of the Declaration of Helsinki and good clinical practice and was approved by the institutional ethical committee (No.15728). All study participants and their parents were given information about the study, and signed consent was obtained from the parents.

\section{Assessment of disease activity}

Disease activity was calculated using the urticaria activity score (UAS) ${ }^{24}$ UASs were determined based on interviews with patients and their families and on physical examination 
findings. The UAS consisted of the sum of the wheal number score and the pruritus score, which ranged from 0-6.

\section{Blood sample collection}

All drugs used by patients were discontinued at least 24 hours before blood sampling. After overnight fasting, peripheral blood samples were collected from an antecubital vein into heparinized tubes; thereafter, blood was centrifuged at $3000 \times \mathrm{g}$ for $10 \mathrm{~min}$ to obtain the plasma. The separated plasma was frozen immediately at $-80^{\circ} \mathrm{C}$ until further analysis of plasma $\mathrm{LXA}_{4}$ levels. Blood samples from the patient and control groups were taken at the same time each day to avoid any possible diurnal variation of LXA4 levels.

\section{Laboratory investigations}

The following tests were routinely performed in all cases: a complete blood count, a liver function test, a thyroid-stimulating hormone test, free thyroxine test, an anti-thyroid peroxidase test, an anti-thyroglobulin antibody test, a total immunoglobulin E test, and a microscopic investigation of stool for parasites.

Atopic status was defined as at least one positive reaction to 10 common aero-allergens using a skin prick test. Standard commercial allergen solutions and lancets (Stallergenes ${ }^{\circledR}$ and Stallerpoint ${ }^{\circledR}$, Paris, France) were used for the skin prick tests. The inhalant panel consisted of Dermatophagoides pteronyssinus, Dermatophagoides farinae, a grass pollen mixture, a weed pollen mixture, an aspergillus mixture, alternaria, cypress, birch, dog epithelia, and cat epithelia. The skin prick test was accepted positive with the existence of a wheal of at least $3 \mathrm{~mm}$ maximum diameter after subtracting the negative value. An autologous serum skin test (ASST) was conducted following the procedure recommended by Sabroe et al. ${ }^{25}$. We were not able to perform skin prick tests and ASSTs on 10 patients because their antihistamine-containing treatments could not be discontinued due to the intensity of their symptoms.

\section{Measurement of plasma $\mathrm{LXA}_{4}$ levels}

$\mathrm{LXA}_{4}$ plasma levels were assessed using a human LXA $_{4}$ enzyme linked immunosorbent assay (ELISA) kit (Shanghai Sunred Biological Technology Co., Shanghai, China). Samples were thawed at room temperature and then centrifuged at $3000 \mathrm{rpm}$ for $10 \mathrm{~min}$. The samples were then diluted to obtain the appropriate concentrations, and an ELISA was performed according to the manufacturer's instructions. The minimal detection limit was $1 \mathrm{ng} / \mathrm{ml}$, and assay sensitivity was $0.52 \mathrm{ng} / \mathrm{ml}$.

\section{Statistical analyses}

A Shapiro-Wilk test was used to test distributions for normality. Parametric data were expressed as the mean \pm standard deviation (SD), and non-parametric data were expressed as the median, interquartile range (IQR). A Mann-Whitney $U$ test was used to calculate the differences between two parameters in the groups. The correlation between two variables was assessed using the Spearman rank correlation coefficient. Categorical data were evaluated using the chi square test; $\mathrm{p}<0.05$ was accepted as statistically significant. Statistical analyses were performed using IBM SPSS 19 (IBM, Chicago, IL, USA).

\section{Results}

The study group consisted of 25 boys and 17 girls, and the control group consisted of 13 boys and 12 girls. The mean ages of the CSU patients and the control group participants were $11.0 \pm 4.2$ and $9.5 \pm 3.9$ years, respectively. No significant differences in age or gender existed between the groups $(p>0.05)$. Liver function tests and thyroid-stimulating hormone and free thyroxin levels were within normal limits, and anti-thyroid peroxidase and anti-thyroglobulin antibody tests and stool investigations for parasites were negative for all patients.

The median value of UASs was 3.0 (2.8-5), and there was no statistically significant difference between ASST-positive and ASSTnegative patients $(p>0.05)$. Some demographic and clinical features of the patients are shown in Table I.

The median value of plasma $\mathrm{LXA}_{4}$ was significantly lower in the patient group compared to the control group $(\mathrm{ng} / \mathrm{ml}$, median [IQR], 60.8 [48.1-71.8] vs. 137.4 [121.4-150.8]). The difference between the groups was statistically significant $(p<0.001$; Fig. 1). Additionally, median [IQR] plasma $\mathrm{LXA}_{4}$ levels were 45.8 $\mathrm{ng} / \mathrm{ml}$ [36.7-67.6] in the ASST-positive CSU patients and $63.8 \mathrm{ng} / \mathrm{ml}$ [58.3-78.9] in the ASST-negative CSU patients. There was also 
a statistically significant difference between these two groups $(p<0.05$; Fig. 2). Despite the negative relationship between the $\mathrm{LXA}_{4}$ levels and UASs, a correlation analysis did not show any statistical significance ( $p>0.05$; Fig. 3 ). Plasma $\mathrm{LXA}_{4}$ levels did not vary between the groups in terms of gender, presence or absence of angioedema and atopic status ( $p>0.05)$. Also, there was not a correlation between plasma Ig $E$ and $\mathrm{LXA}_{4}$ levels $(\mathrm{p}>0.05)$.

\section{Discussion}

Our study results showed that patients with CSU have obviously reduced plasma $\mathrm{LXA}_{4}$ levels compared to healthy controls and that ASST-positive patients' $\mathrm{LXA}_{4}$ levels were even lower than those of ASST-negative patients. These results highlight the possible role of $\mathrm{LXA}_{4}$ in the pathogenesis of CSU for the first time in the literature. Although there was a negative relationship between plasma $\mathrm{LXA}_{4}$ levels and UASs, a correlation analysis did not show a statistical significance $(\mathrm{p}>0.05)$. We chose to use UAS instead of UAS7 (sum of UAS scores for seven consecutive days) in this study because $\mathrm{LXA}_{4}$ has a short half-life and we aimed to compare instant UAS and plasma LXA $_{4}$ levels as much as possible ${ }^{12}$. Although UAS is the only validated tool for assessing the disease activity of CSU, 2,24 it has some limitations. For example, it includes subjective interpretations of families or patients and reflects disease activity in the last 24 hours instead of momentary activity. These limitations and the small sample size may be the reasons the correlation analysis did not result in significance. However, it is challenging to be able to create a larger pediatric patient group when the disease being investigated is CSU because the prevalence in children is only one-tenth of that in adults.3,26,27

Asthma is the best studied disease that shows the role of lipoxinA4 in allergic inflammation. LXs can be demonstrated in sputum and bronchoalveolar lavage fluids of asthmatic patients. ${ }^{6}$ LXs block airway responsiveness, reduce airway inflammation, reduce granulocyte infiltration to airways, lower the number of inflammatory cells and the levels of proinflammatory mediators, such as cysteinyl leukotrienes, and decrease oxidative stress. ${ }^{28-30}$ Gagliardo et al. ${ }^{31}$ showed defective $\mathrm{LXA}_{4}$ generation and FPR2/ALX receptor expression in children with severe asthma. Karra and co-workers reported that $\mathrm{LXB}_{4}$ significantly reduces nasal mucosal leukocytes, eosinophil chemotaxis, and degranulation of mast cells in a murine model of allergic rhinitis. ${ }^{20} \mathrm{Wu}$ at al. ${ }^{23}$ demonstrated that topical application of 15(R/S)-methyl-LXA , a LXA $_{4}$ agonist, is well tolerated, has no side effects, and significantly improves all features of atopic dermatitis. The anti-inflammatory effect of $15(\mathrm{R} / \mathrm{S})$-methyl$\mathrm{LXA}_{4}$ cream was found to be similar to that of mometasone cream in this study.

Reduced $\mathrm{LXA}_{4}$ biosynthesis in CSU patients, which we identified in our study, can explain the vast majority of what we know about the pathogenesis of CSU. Wheals, flares, and angioedema, which are characteristic of CSU, arise as a result of increased vascular permeability and extravascular leakage of intravascular fluid and proteins. ${ }^{32}$ In their studies, Ereso at al. ${ }^{33}$ and Pang et al. ${ }^{34}$ determined that $\mathrm{LXA}_{4}$ decreases vascular hyperpermeability and microvascular fluid leakage.

Neutrophils are the main inflammatory cells that compose the cellular infiltrates of urticarial plaque, and they exist within one hour after stimulus in the urticarial wheal. ${ }^{4,35} \mathrm{LXA}_{4}$ inhibits chemotaxis, adhesion, transmigration, and cytokine production of neutrophils. ${ }^{7}$ Eosinophils are also involved and are located mainly in the perivascular dermis. ${ }^{35}$ According to researchers who state that the coagulation cascade is activated in CSU, eosinophil activation is an initial event and triggers urticarial episodes. ${ }^{36} \mathrm{LXA}_{4}$ inhibits chemotaxis, interleukin (IL)-5, and eotaxin secretion of eosinophils. ${ }^{7}$ Additionally, $\mathrm{LXA}_{4}$ can block granulocyte-macrophage colonystimulating factor-induced activation signaling in eosinophils, which is one of the crucial cytokines affecting eosinophil activation and survival. ${ }^{37,38}$ Moreover, $\mathrm{LXA}_{4}$ induces NK cell-induced apoptosis of both eosinophils and neutrophils. ${ }^{39}$ Mast cell numbers remain unaltered in patients with CSU and are similar in uninvolved skin and healthy control subjects. ${ }^{40}$ However, inappropriate activation and degranulation of mast cells are key pathophysiological events. ${ }^{4}$ Two previous studies reported that $\mathrm{LXA}_{4}$ is a potent inhibitor of immunoglobulin (Ig) E-mediated mast cell 
degranulation in a dose-dependent manner. ${ }^{20,41}$

An autoimmune origin has become the most accepted hypothesis in recent years. ${ }^{4}$ Functional autoantibodies in the sera of CSU patients have been demonstrated against IgE and FceRIa. ${ }^{42}$ In our study, ASST-positive patients had reduced plasma $\mathrm{LXA}_{4}$ levels compared to ASST-negative patients. This may be partly explained in two ways; (1) FceRI signaling is transmitted intracellularly by the NF- $\mathrm{BB}$ pathway in mast cells. ${ }^{43}$ Additionally, activation and cytokine production is mainly dependent on this pathway. ${ }^{43-45}$ (2) LXA $_{4}$ decreases immunoglobulin production on memory B-cells in a dose-dependent manner. ${ }^{15} \mathrm{LXA}_{4}$ can reduce both autoantibody secretion and FceRI signaling via NF- $\kappa B$ inhibition and in this way may play a more dominant role in CSU with autoimmune origins.

Although elevated levels by themselves cannot explain the entire disease pathogenesis, the increased production of some cytokines, chemokines, or metabolites-including IL-6, vascular endothelial growth factor (VEGF), matrix metallopeptidase 9 (MMP-9), and reactive oxygen species-was reported in previous clinical studies. ${ }^{46-49}$ LXs can inhibit the generation of pro-inflammatory cytokines and chemokines via the inhibition of transcription factors, such as NF- $\kappa \mathrm{B}$ and activator protein 1. ${ }^{50-52}$ In this regard, several in vitro studies and animal models have proven that $\mathrm{LXA}_{4}$ and its analogues can decrease IL- 6 secretion, ${ }^{6}$ MMP-9 expression, ${ }^{53}$ and VEGF expression ${ }^{54}$ and can block ROS generation. ${ }^{55}$

Various LX analogues have developed because of the rapid inactivation and short half-life of natural LXs. ${ }^{12}$ Promising therapeutic bioactions of LX analogues have been determined at several experimental disease models, including atopic dermatitis, contact dermatitis, and asthma. ${ }^{12,19,56}$ Additionally, clinical studies of allergic dermatoses have reported comparable therapeutic efficacy of topical corticosteroids but without any side effects. ${ }^{19,23}$ If further studies also demonstrate reduced $\mathrm{LXA}_{4}$ levels in CSU patients, this disease may be another research area for investigating the possible therapeutic importance of these agents.

In conclusion, plasma $\mathrm{LXA}_{4}$ levels are significantly decreased in pediatric patients with CSU compared to healthy controls. Additionally,
ASST-positive patients have even lower values than ASST-negative patients. Deficient LXA $_{4}$ bioactions at the cellular level can explain many aspects of the CSU pathogenesis puzzle that we know but we cannot combine. There are many lipoxin analogues offering new and attractive treatment options. We emphasize that this is the first study conducted on this topic, and that further studies are needed. Nevertheless, proresolving eicosanoids may be the answer to many questions about the etiopathogenesis of CSU.

\section{REFERENCES}

1. Kaplan AP, Greaves M. Pathogenesis of chronic urticaria. Clin Exp Allergy 2009; 39: 777-787.

2. Zuberbier T, Aberer W, Asero R, et al.; European Academy of Allergy and Clinical Immunology; Global Allergy and Asthma European Network; European Dermatology Forum; World Allergy Organization. The EAACI/GA(2) LEN/EDF/WAO Guideline for the definition, classification, diagnosis, and management of urticaria: the 2013 revision and update. Allergy 2014; 69: $868-887$.

3. Powell RJ, Leech SC, Till S, Huber PA, Nasser SM, Clark AT; British Society for Allergy and Clinical Immunology. BSACI guideline for the management of chronic urticaria and angioedema. Clin Exp Allergy 2015; 45: 547-565.

4. Jain S. Pathogenesis of chronic urticaria: an overview. Dermatol Res Pract 2014; 2014: 674709.

5. $\mathrm{Xu} \mathrm{Y,} \mathrm{Chen} \mathrm{G.} \mathrm{Mast} \mathrm{cell} \mathrm{and} \mathrm{autoimmune} \mathrm{diseases.}$ Mediators Inflamm 2015; 2015: 246126.

6. Levy BD, Vachier I, Serhan CN. Resolution of inflammation in asthma. Clin Chest Med 2012; 33: 559-570.

7. Romano M, Cianci E, Simiele F, Recchiuti A. Lipoxins and aspirin-triggered lipoxins in resolution of inflammation. Eur J Pharmacol 2015; 760: 49-63.

8. Serhan $\mathrm{CN}$. Novel lipid mediators and resolution mechanisms in acute inflammation: to resolve or not? Am J Pathol 2010; 177: 1576-1591.

9. Levy BD, Vachier I, Serhan CN. Resolution of inflammation in asthma. Clin Chest Med 2012; 33: 559-570.

10. Serhan CN. Resolution phase of inflammation: novel endogenous anti-inflammatory and proresolving lipid mediators and pathways. Annu Rev Immunol 2007; 25: 101-137.

11. Higgins G, Ringholz F, Buchanan P, McNally P, Urbach V. Physiological impact of abnormal lipoxin $\mathrm{A}_{4}$ production on cystic fibrosis airway epithelium and therapeutic potential. Biomed Res Int 2015; 2015: 781087.

12. Maderna P, Godson C. Lipoxins: resolutionary road. Br J Pharmacol 2009; 158: 947-959. 
13. Chinthamani S, Odusanwo O, Mondal N, Nelson J, Neelamegham S, Baker OJ. Lipoxin A4 inhibits immune cell binding to salivary epithelium and vascular endothelium. Am J Physiol Cell Physiol 2012; 302: C968-C978.

14. Levy BD, Lukacs NW, Berlin AA, et al. Lipoxin A4 stable analogs reduce allergic airway responses via mechanisms distinct from CysLT1 receptor antagonism. FASEB J 2007; 21: 3877-3884.

15. Ramon S, Bancos S, Serhan CN, Phipps RP. Lipoxin $\mathrm{A}_{4}$ modulates adaptive immunity by decreasing memory B-cell responses via an ALX/FPR2-dependent mechanism. Eur J Immunol 2014; 44: 357-369.

16. Herrera BS, Kantarci A, Zarrough A, Hasturk H, Leung KP, Van Dyke TE. LXA4 actions direct fibroblast function and wound closure. Biochem Biophys Res Commun 2015; 464: 1072-1077.

17. Petri MH, Laguna-Fernandez A, Tseng CN, Hedin U, Perretti M, Bäck M. Aspirin-triggered 15-epi-lipoxin $\mathrm{A}_{4}$ signals through FPR2/ALX in vascular smooth muscle cells and protects against intimal hyperplasia after carotid ligation. Int J Cardiol 2015; 179: 370-372.

18. Serhan CN. Lipoxins and aspirin-triggered 15-epilipoxin biosynthesis: an update and role in antiinflammation and pro-resolution. Prostaglandins Other Lipid Mediat 2002; 68-69: 433-455.

19. Schottelius AJ, Giesen C, Asadullah K, et al. An aspirin-triggered lipoxin A4 stable analog displays a unique topical anti-inflammatory profile. J Immunol 2002; 169: 7063-7070.

20. Karra L, Haworth O, Priluck R, Levy BD, Levi-Schaffer F. Lipoxin $\mathrm{B}_{4}$ promotes the resolution of allergic inflammation in the upper and lower airways of mice. Mucosal Immunol 2015; 8: 852-862.

Vachier I, Bonnans C, Chavis C, et al. Severe asthma is associated with a loss of LX4, an endogenous antiinflammatory compound. J Allergy Clin Immunol 2005; 115: 55-60.

21. Tahan F, Saraymen R, Gumus H. The role of lipoxin A4 in exercise-induced bronchoconstriction in asthma. J Asthma 2008; 45: 161-164.

22. Wu SH, Chen XQ, Liu B, Wu HJ, Dong L. Efficacy and safety of $15(\mathrm{R} / \mathrm{S})$-methyl-lipoxin $\mathrm{A}(4)$ in topical treatment of infantile eczema. Br J Dermatol 2013; 168: $172-178$

23. Zuberbier T, Asero R, Bindslev-Jensen C, et al; Dermatology Section of the European Academy of Allergology and Clinical Immunology; Global Allergy and Asthma European Network; European Dermatology Forum; World Allergy Organization. EAACI/GA(2) LEN/EDF/WAO guideline: management of urticaria. Allergy 2009; 64: 1427-1443.

24. Sabroe RA, Grattan CE, Francis DM, Barr RM, Kobza Black A, Greaves MW. The autologous serum skin test: a screening test for autoantibodies in chronic idiopathic urticaria. Br J Dermatol 1999; 140: 446-452.

25. Maurer M, Weller K, Bindslev-Jensen C, et al. Unmet clinical needs in chronic spontaneous urticaria. A GA $^{2}$ LEN task force report. Allergy 2011; 66: 317-330.
26. Khakoo G, Sofianou-Katsoulis A, Perkin MR, Lack G. Clinical features and natural history of physical urticaria in children. Pediatr Allergy Immunol 2008; 19: 363-366.

27. Levy BD, De Sanctis GT, Devchand PR, et al. Multipronged inhibition of airway hyper-responsiveness and inflammation by lipoxin A(4). Nat Med 2002; 8: 1018-1023.

28. Levy BD, Clish CB, Schmidt B, Gronert K, Serhan $\mathrm{CN}$. Lipid mediator class switching during acute inflammation: signals in resolution. Nat Immunol 2001; 2: 612-619.

29. Ono E, Dutile S, Kazani S, et al; National Heart, Lung, and Blood Institute's Asthma Clinical Research Network. Lipoxin generation is related to soluble epoxide hydrolase activity in severe asthma. Am J Respir Crit Care Med 2014; 190: 886-897.

30. Gagliardo R, Gras D, La Grutta S, et al. Airway lipoxin A4/formyl peptide receptor 2-lipoxin receptor levels in pediatric patients with severe asthma. J Allergy Clin Immunol 2016; 137: 1796-1806.

31. Carr TF, Saltoun CA. Chapter 21: Urticaria and angioedema. Allergy Asthma Proc 2012; 33(Suppl 1):S70-S72

32. Ereso AQ, Cureton EL, Cripps MW, et al. Lipoxin a(4) attenuates microvascular fluid leak during inflammation. J Surg Res 2009; 156: 183-188.

33. Pang HY, Huang YP, Liu ZJ, et al. Effect of lipoxin A (4) on lipopolysaccharide-induced endothelial hyperpermeability in human umbilical vein endothelial cell. Zhonghua Fu Chan Ke Za Zhi 2011; 46: 199-204.

34. Caproni M, Giomi B, Volpi W, et al. Chronic idiopathic urticaria: infiltrating cells and related cytokines in autologous serum-induced wheals. Clin Immunol 2005; 114: 284-292.

36. Asero R, Tedeschi A, Marzano AV, Cugno M. Chronic spontaneous urticaria: immune system, blood coagulation, and more. Expert Rev Clin Immunol 2016; 12: 229-231.

37. Starosta V, Pazdrak K, Boldogh I, Svider T, Kurosky A. Lipoxin A4 counterregulates GM-CSF signaling in eosinophilic granulocytes. J Immunol 2008; 181: 8688-8699.

38. Pazdrak K, Young TW, Stafford S, et al. Crosstalk between ICAM-1 and granulocyte-macrophage colony-stimulating factor receptor signaling modulates eosinophil survival and activation. J Immunol 2008; 180: 4182-4190.

39. Barnig C, Cernadas M, Dutile S, et al. Lipoxin A4 regulates natural killer cell and type 2 innate lymphoid cell activation in asthma. Sci Transl Med 2013; 5: 174 ra26.

40. Smith CH, Kepley C, Schwartz LB, Lee TH. Mast cell number and phenotype in chronic idiopathic urticaria. J Allergy Clin Immunol 1995; 96: 360-364.

41. Martin N, Ruddick A, Arthur GK, et al. Primary human airway epithelial cell-dependent inhibition of human lung mast cell degranulation. PLoS One 2012; 7: e43545. 
42. Konstantinou GN, Asero R, Ferrer M, et al. EAACI taskforce position paper: evidence for autoimmune urticaria and proposal for defining diagnostic criteria. Allergy 2013; 68: 27-36.

43. Klemm S, Ruland J. Inflammatory signal transduction from the Fc epsilon RI to NF-kappa B. Immunobiology 2006; 211: 815-820.

44. Peng Y, Power MR, Li B, Lin TJ. Inhibition of IKK down-regulates antigen + IgE-induced TNF production by mast cells: a role for the IKK-IkappaB-NF-kappaB pathway in IgE-dependent mast cell activation. J Leukoc Biol 2005; 77: 975-983.

45. Marquardt DL, Walker LL. Dependence of mast cell IgE-mediated cytokine production on nuclear factorkappaB activity. J Allergy Clin Immunol 2000; 105: 500-505.

46. Kasperska-Zajac A, Grzanka A, Damasiewicz-Bodzek A. IL-6 Transsignaling in Patients with Chronic Spontaneous Urticaria. PLoS One. 2015; 10: e0145751.

47. Dilek F, Ozceker D, Ozkaya E, et al. Plasma Levels of Matrix Metalloproteinase-9 in Children With Chronic Spontaneous Urticaria. Allergy Asthma Immunol Res 2016; 8: 522-526.

48. Tedeschi A, Asero R, Marzano AV, et al. Plasma levels and skin-eosinophil-expression of vascular endothelial growth factor in patients with chronic urticaria. Allergy 2009; 64: 1616-1622.

49. Dilek F, Ozceker D, Ozkaya E, et al. Oxidative stress in children with chronic spontaneous urticaria. Oxid Med Cell Longev 2016; 2016: 3831071.
50. Wu SH, Lu C, Dong L, Zhou GP, He ZG, Chen ZQ. Lipoxin A4 inhibits TNF-alpha-induced production of interleukins and proliferation of rat mesangial cells. Kidney Int 2005; 68: 35-46.

51. József L, Zouki C, Petasis NA, Serhan CN, Filep JG. Lipoxin A4 and aspirin-triggered 15-epi-lipoxin A4 inhibit peroxynitrite formation, NF-kappa B and AP-1 activation, and IL-8 gene expression in human leukocytes. Proc Natl Acad Sci U S A 2002; 99: 13266 13271.

52. Macdonald LJ, Boddy SC, Denison FC, Sales KJ, Jabbour HN. A role for lipoxin $A_{4}$ as an anti-inflammatory mediator in the human endometrium. Reproduction 2011; 142: 345-352.

53. Wu Y, Wang YP, Guo P, et al. A lipoxin A4 analog ameliorates blood-brain barrier dysfunction and reduces MMP-9 expression in a rat model of focal cerebral ischemia-reperfusion injury. J Mol Neurosci 2012; 46: 483-491.

54. Medeiros R, Rodrigues GB, Figueiredo CP, et al. Molecular mechanisms of topical anti-inflammatory effects of lipoxin A(4) in endotoxin-induced uveitis. Mol Pharmacol 2008; 74: 154-161.

55. Nascimento-Silva V, Arruda MA, Barja-Fidalgo C Fierro IM. Aspirin-triggered lipoxin A4 blocks reactive oxygen species generation in endothelial cells: a novel antioxidative mechanism. Thromb Haemost 2007; 97: 88-98.

56. Levy BD, Lukacs NW, Berlin AA, et al. Lipoxin A4 stable analogs reduce allergic airway responses via mechanisms distinct from CysLT1 receptor antagonism. FASEB J 2007; 21: 3877-3884. 